\title{
Clinical Guidelines For The Management Of Cutaneous Leishmaniasis In British Military Personnel
}

\author{
MS Bailey, AD Green, CJ Ellis, TJ O’Dempsey, NJ Beeching, \\ DN Lockwood, PL Chiodini, AD Bryceson
}

Maj MS Bailey RAMC Specialist Registrar in Infectious Diseases and Tropical Medicine, AMD, Slim Road, Camberley, Surrey, GU15 4NP

Wg Cdr AD Green RAF Tri-Service Consultant in Communicable

Disease Control,

Defence Medical

Services Department,

Whitehall, London,

SW1A 2HB

\section{Dr CJ Ellis}

Honorary Civilian Consultant Adviser in

Infectious Diseases,

Birmingham Heartlands

Hospital, Bordesley

Green East,

Birmingham, B9 5SS

Dr TJ O'Dempsey,

Dr NJ Beeching

Consultant Physicians

in Infectious Diseases

and Tropical Medicine,

Liverpool School of

Tropical Medicine,

Pembroke Place,

London, L3 5QA

Dr DN Lockwood,

Prof PL Chionini,

Prof AD Bryceson

Consultant Physicians

in Infectious Diseases

and Tropical Medicine,

Hospital for Tropical

Diseases, Mortimer

Market, Capper Street,

London, WC1E 6AU

\section{Introduction}

Cutaneous leishmaniasis (CL) is an increasing problem for British military personnel due to current deployments in Belize, Iraq and Afghanistan. Recent developments in the diagnosis and treatment of CL have emphasised the importance of a speciesspecific approach to management (1). This is especially important in New World CL (NWCL), where there may be a risk of mucocutaneous leishmaniasis (MCL) developing.

These guidelines are primarily written for the management of military patients. They differ from civilian patients because having $\mathrm{CL}$ will usually prevent them from carrying out their duties and so there must be a greater emphasis on active treatment. These guidelines are not intended to replace Surgeon General's Policy Letter (SGPL) 09/04 (2), but aim to establish clinical standards for the management of suspected CL by physicians. There are currently no plans nor any need for investigation or treatment of suspected cases to take place on overseas deployments. Any suspicious lesions in personnel deployed overseas should be discussed with an appropriate specialist (Appendix 1) and the individual affected must be evacuated for investigation and treatment if advised.

The guidelines are summarised in Figure 1, but the following text should also be read in full.

\section{Suspicious Lesions}

A suspicious lesion is defined as any skin nodule or ulcer with a raised edge that occurs during or within 2 years of travel to a leishmaniasis endemic area, which does not resolve with 1-2 weeks of appropriate antibacterial therapy. Leishmaniasis endemic areas may be identified from the relevant military Medical Intelligence Assessment (MIA) or from www.who.int/leishmaniasis. Suspected cases may be discussed with the clinical staff listed in Appendix 1 and digital photographs (taken with written consent) can be extremely useful in this respect. Military patients should be notified to the relevant communicable disease control appointment by the referring medical officer at this stage (2).

\section{Referral to a Specialist}

All patients with suspected CL should be referred as soon as possible, even before they have completed their initial course of antibacterial therapy. Patients should still be referred if their lesions appear static or even if they are improving. It is not necessary for patients to see a dermatologist before being referred. If investigations are negative, then patients will be referred on to a dermatologist at their local hospital.

These guidelines are not intended to exclude any hospital specialists who are already experienced in the complexities of investigating and treating CL. However, management should involve species identification and species-specific treatment wherever possible. This will require liaison with one of the laboratories listed in Appendix 2 and hence it may be expedient to refer patients to the clinical facilities at one of these locations. Military patients must be referred to one of the specialists listed in Appendix 1 (2).

\section{Sampling of Lesions}

A rigorous approach to making a parasitological diagnosis is important because of the toxicity associated with systemic sodium stibogluconate therapy, which remains the mainstay of treatment in these guidelines. Suspicious lesions should be sampled by aspiration (without dilution), scraping (either directly from an ulcer or from skin slits) and biopsy (usually with a $4 \mathrm{~mm}$ punch biopsy tool). Skin biopsy is the most useful of these methods, but lesions that can not be biopsied (eg. on a finger, ear or face) should still be aspirated and scraped.

Sampling should only be performed by staff who are properly trained in these techniques, otherwise poor results and complications may occur. Additional descriptions of these sampling methods are available elsewhere (3). It is recommended that contact be made with one of the laboratories listed in Appendix 2 before any sampling is performed.

A lesion that has re-epithelialised would usually not be sampled. If a lesion is reported to be improving with evidence of a wellformed / persistent scab covering any ulceration and minimal inflammation at its edge, then sampling is still performed. However, this approach will be reviewed when future results are available.

If the initial sampling yields negative 


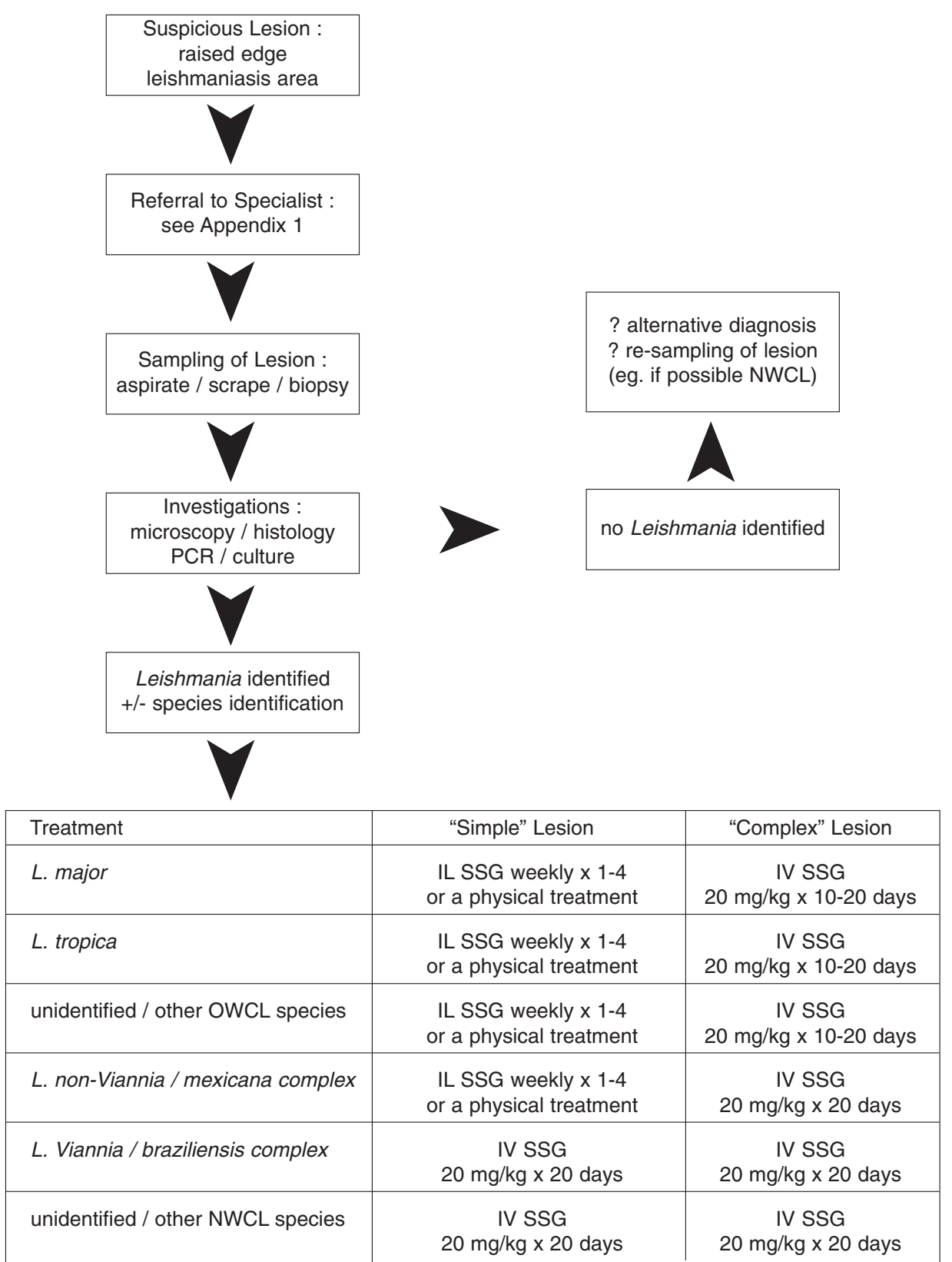

Fig 1. Guidelines for the management of military patients.

results and the lesion is worsening or not improving, then sampling should be repeated. If initial sampling yields negative results and the lesion is improving and Old World CL (OWCL) is suspected, then sampling is usually not repeated. However, if the initial sampling yields negative results and the lesion is improving and New World CL (NWCL) is suspected, then sampling should still be repeated. Overall, there should be a lower threshold for sampling and re-sampling of suspected NWCL lesions because of the risk of MCL developing in the future.

\section{Aspiration}

Aspirations are performed from the raised edge or nodular part of a lesion. A $0.5 \mathrm{~mm}$ diameter needle is tightly connected to an empty syringe. The needle is slowly advanced, with negative pressure applied, in a straight line (to avoid blood contamination) along the edge of an ulcer or into the centre of a solid lesion. It is withdrawn in a similar manner, taking care not to draw any air into the syringe. The needle is then disconnected, air is drawn into the syringe, the needle is reattached and the contents are blown out rapidly onto a clean, polished and alcoholfree microscope slide. The aspirate should be gently spread on the slide using the tip of the needle to achieve best results on microscopy. This slide should be labelled to identify the patient and the sampling technique used and transported as directed by the laboratory. Aspirates may also be mixed into a tube of PCR buffer and transported as directed or else it may be possible to perform PCR tests on the material sent as a slide.

\section{Scraping}

Scrapings are performed either from the surface of an ulcer or from skin slits. Ulcer scrapings should be taken from both the edge and the centre of the lesion. Slit skin 
scrapings should be taken from the raised edge or nodular part of a lesion. Either type of scraping should be smeared onto a clean, polished and alcohol-free microscope slide. This slide should be labelled to identify the patient and the sampling technique used and transported as directed by the laboratory. Scrapings may also be mixed into a tube of PCR buffer and transported as directed or else it may be possible to perform PCR tests on the material sent as a slide.

\section{Biopsy}

Two standard $4 \mathrm{~mm}$ punch biopsies from the raised edge or nodular part of a lesion should usually be performed. Blood contamination can be prevented by using local anaesthetic with adrenaline (where anatomicallypermitted), applying pressure haemostasis before removing the core and gently rolling the core on gauze after its removal. The first biopsy is cut lengthways into 2 equal pieces and used as follows. The freshly-cut surface of one piece is firmly pressed (not smeared) to create a series of 3-5 touch preparations on a clean, polished and alcohol-free microscope slide, which is labelled to identify the patient and the sampling technique used and transported as directed. This piece is then put into an appropriate medium (either PCR buffer, normal saline or viral culture medium containing anti-bacterial, but no antifungal additives) for PCR testing and transported as directed by the laboratory. The other piece is placed directly into a tube of culture medium and transported as directed by the laboratory. The second biopsy should be handled gently to prevent any crush artefacts, put into formalin for histological examination and transported as directed by the laboratory.

\section{Investigations}

It is recommended that contact be made with the nearest laboratory listed in Appendix 2 before any samples are sent and before any investigations are performed.

\section{Microscopy}

Slides must be prepared immediately from aspirates, scrapings or touch preparations of skin biopsies in all suspected cases of CL. These should be air-dried (and preferably fixed in methanol for 1 minute) and sent to one of the laboratories listed in Appendix 2. (Where they will be stained with Giemsa at $\mathrm{pH} 6.8$ and examined for the presence of Leishmania amastigotes.) It may also be possible to perform PCR analysis on slide material, but a dedicated sample for this purpose will achieve better results.

\section{Histology}

Skin biopsy tissue is prepared and stained with either Haemotoxylin \& Eosin or Giemsa. Most pathology laboratories will be able to identify Leishmania amastigotes so long as they are alerted to this possibility. However, it is recommended that all histology specimens are also forwarded for review to Professor Sebastian Lucas, Department of Histopathology, St Thomas's Hospital, London, SE1 7EH (email : sebastian.lucas@kcl.ac.uk / telephone : 020 7188 2954), who has confirmed that there will be no charge for this service.

\section{PCR Testing}

Samples that are negative on microscopy may undergo a generic / screening PCR test for parasites of the Leishmania genus $(4,5)$ before they undergo any species-specific PCR tests. Unless specimens are negative on both microscopy and the generic / screening PCR test, then they should undergo further PCR tests dependant on whether OWCL or NWCL is suspected. If OWCL is suspected then a species-specific PCR test will be performed to identify L. major, L. tropica, or L. donovani complex species (6). If NWCL is suspected then a specific PCR test for parasites of the L. Viannia sub-genus ( $L$. braziliensis complex) will be performed (7 or 8,9 ).

These tests are only available at the laboratories listed in Appendix 2 and they should be contacted for advice on what samples to send and how to transport them. PCR tests can be performed on material preserved in paraffin blocks or even from microscopy slides if no other tissue is available. This option should also be discussed with the laboratory in question.

\section{Culture}

Specimens for culture are placed in either semi-solid blood agar or a modified NoveyMacNeal-Nicolle (NNN) medium and incubated at an appropriate temperature. In practice it will only be possible to perform this investigation for patients seen at either the Liverpool School of Tropical Medicine (LSTM) or the Hospital for Tropical Diseases (HTD). Although PCR speciation tests are sufficient for clinical practice, culture remains an important technique and the only way of obtaining sufficient parasites for subspeciation tests, such as zymodeme analysis.

\section{Other Investigations}

Existing serology tests have no role in the diagnosis of cutaneous leishmaniasis.

\section{Treatment}

Recommended treatments are summarised in the table within Figure 1.

"Simple" vs. "Complex" Lesions

"Complex" lesions are defined as those that:

- are $>2-3$ in number

- are $>40 \mathrm{~mm}$ in their maximum diameter 
- are associated with lymphatic or lymph node involvement

- could lead to cosmetic problems eg. on the face, ear or neck

- could lead to functional problems eg. on the hand or over a joint

- have not responded adequately to treatment as a "simple" lesion

\section{Observation}

Suspected or confirmed OWCL lesions that are improving may be simply observed, especially if they are due to L. major. OWCL due to L. tropica or L. donovani complex species is likely to be more prolonged and to need treatment. NWCL lesions that are improving may only be observed if the cause is proven to be non-Viannia (L. mexicana complex) parasites. NWCL lesions due to L. Viannia sub-genus (L. braziliensis complex) parasites or confirmed NWCL lesions for which a species can not be identified must always receive systemic therapy because of the risk of MCL developing in the future. Suspected NWCL lesions that are improving and remain negative for all Leishmania investigations should be observed, but patients must be warned of the $\sim 5 \%$ risk of MCL developing in the future and advised to seek medical attention for any oro-nasal symptoms or genital ulceration.

\section{Physical Treatments}

Cryotherapy, thermotherapy or curettage may be used for "simple" OWCL lesions and "simple" non-Viannia NWCL lesions (ie. "simple" CL lesions that do not have the potential to become MCL). However, lack of equipment and expertise plus concerns over altered pigmentation, secondary infection, dissemination of parasites, slower healing and larger scars mean that alternative treatments are usually preferred. More recent versions of certain physical treatments do offer some hope for the future (10).

\section{Intra-Lesional (IL) Sodium \\ Stibogluconate (SSG)}

This is a more established treatment for "simple" CL lesions that do not have the potential to become MCL. Correct technique is essential and this is well described elsewhere (1). However, some patients are unable to tolerate the transient pain of infiltration and it will be less effective for atypical infections with unexpected systemic features (11). The advantages of IL SSG are that it does not require hospital admission, monitoring investigations or restriction of exercise. However, sometimes complete healing may be quicker and relapse/recurrence may be less likely with IV SSG. Hence an individual assessment for each patient is necessary and these matters will be studied further.

\section{Intra-Venous (IV) Sodium Stibogluconate (SSG)}

There is now a consensus that $20 \mathrm{mg} / \mathrm{kg}$ (with no upper limit) for 20 days should be the standard dose of IV SSG for NWCL (12) despite the associated toxicity (13). The situation for OWCL is less clear, but there is some evidence that $20 \mathrm{mg} / \mathrm{kg}$ (with no upper limit) for 10 days may be sufficient according to recent and ongoing trials in the USA (14). OWCL lesions that are not clearly responding at 10 days should be treated for 20 days in total. An observational study of the efficacy and toxicity resulting from these regimens will be performed.

IV SSG may be given via a series of butterfly needles or intravenous cannulae, which should be changed frequently to avoid phlebitis. It may also be given via a peripherally-inserted central catheter (PICC) line, but this is associated with a relatively high complication rate (15). IV SSG should be diluted in 5\% glucose and administered over 30 minutes. Filtration, cardiac monitoring and keeping the infusion in the dark are not necessary. Patients receiving IV SSG do not need to be admitted, but should be advised to avoid exercise and alcohol for the duration of their treatment and for 1 week afterwards.

Adverse effects with IV SSG at $20 \mathrm{mg} / \mathrm{kg}$ (with no upper limit) are common (13) and patients should undergo twice weekly monitoring investigations, including a full blood count (FBC), urea \& electrolytes (U\&E), liver function tests (LFT), serum amylase and electrocardiographs (ECG). It is recommended that treatment be interrupted if :

- FBC changes become significantly abnormal

- U\&E changes become significantly abnormal

- liver transaminases exceed 15 times the upper limit of normal (ULN)

- serum amylase exceeds 4 times the ULN or if other features of pancreatitis develop

- ECG changes become significantly abnormal (eg. QTc prolongation > 500 milliseconds)

In practice the most common reason for interrupting treatment is musculoskeletal symptoms, which may be severe (especially in older patients), but often settle with non-steroidal anti-inflammatory drugs (NSAIDs). Treatment can usually be recommenced once the abnormality in question has begun to return to normal.

Military patients treated with IV SSG for 20 days should be given sick leave and advised to avoid exercise and alcohol for 1 week after treatment. They may also require a few weeks of gradually increasing exercise to regain full fitness. 


\section{Other Treatments}

Topical paramomycin remains a muchdebated treatment for CL and there are concerns that lesions may initially get worse on this therapy.

Oral fluconazole has been shown to be effective against $L$. major lesions (16), but our experience has been that lesions may continue to enlarge after treatment has begun. Some studies have shown that oral ketoconazole may also be of benefit in certain forms of CL (17), but further research is required to confirm this.

Oral miltefosine has been used with mixed results in NWCL (18). It performed well against $L$. $V$. panamensis in Colombia, but poorly against $L . V$. braziliensis and $L$. mexicana in Guatemala - which is more comparable to the situation in Belize. Preliminary results from its use against $L$. tropica in Afghanistan are disappointing.

Intramuscular pentamidine is now used for CL amongst French military personnel working in French Guyana (19). However, it has been less successful against different Leishmania species in other South American countries (20). In addition the risk of diabetes mellitus and other adverse effects may outweigh its possible benefits.

Intravenous amphotericin may be effective against CL, but it is not known which of the various preparations (standard, colloidal or liposomal) produces the best results.

\section{Secondary Infections}

Bacterial secondary infections may exacerbate and prolong CL lesions and should be treated with either co-amoxiclav or clindamycin. Patients with lesions that are not fully healed should avoid physical activities that could lead to disruption or secondary infection of their lesions.

\section{Follow-Up Arrangements}

Patients must be reviewed 6 weeks after the commencement of treatment and monthly thereafter until their lesions have fully healed. A final review 6 months after treatment is also recommended and patients should be warned of the possibility of recurrence for up to 2 years.

\section{Audit \& Research}

All confirmed cases of CL (including those with only a clinical diagnosis) should have data recorded as per Appendix 3. Wherever possible these forms should be completed electronically and emailed to Major Mark Bailey RAMC (mark.bailey@ramc.org).This will enable an audit and observational study to be performed on the approach to management recommended in these guidelines. Further studies may follow in due course.

\section{Conclusion}

$\mathrm{CL}$ is a rare, but increasing problem in
British military personnel. Its management requires specialist expertise that is not widely available. These guidelines are not intended to exclude hospital specialists who are already experienced in managing CL. However, a standard approach to management is needed in order to optimise patient care, consolidate current knowledge and learn from future cases. The treatments recommended may seem "conservative" compared to some research findings, but they represent a foundation on which to build in the future. Suspected CL in military personnel must be managed in accordance with these guidelines by one of the specialists listed in Appendix 1.

\section{References}

1. Blum J, Desjeux P, Schwartz E, Beck B, Hatz C. Treatment of cutaneous leishmaniasis among travellers. F Antimicrob Chemotherap 2004; 53: 15866.

2. Surgeon General's Policy Letter. Prevention, Identification, Referral and Follow-Up of Leishmaniasis. SGPL 09/04, Defence Medical Services Department, Ministry of Defence, 2004.

3. Herwaldt BL. Leishmaniasis. The Lancet 1999; 354: 1191-1199.

4. Van Eys GJ, Schoone GJ, Kroon NC, Ebeling SB. Sequence analysis of small subunit ribosomal RNA genes and its use for detection and identification of Leishmania parasites. Mol Biochem Parasitol 1992; 51: $133-42$

5. Meredith SE, Zijlstra EE, Schoone GJ et al. Development and application of the polymerase chain reaction for the detection and identification Leishmania parasites in clinical material. Arch Inst Pasteur Tunis 1993; 70: 419-31.

6. Noyes HA, Reyburn H, Bailey JW, Smith D. A nested-PCR-based schizodeme method for identifying Leishmania kinetoplast minicircle classes directly from clinical samples and its application to the study of the epidemiology of Leishmania tropica in Pakistan. F Clin Microbiol 1998; 36: 2877-81.

7. De Bruijn MH, Barker DC. Diagnosis of New World leishmaniasis: specific detection of species of the Leishmania braziliensis complex by amplification of kinetoplast DNA. Acta Trop 1992; 52: 45-58.

8. Noyes HA, Stevens JR, Teixeira M, Phelan J, Holz P. A nested PCR for the ssrRNA gene detects Trypanosoma binneyi in the platypus and Trypanosoma sp. in wombats and kangaroos in Australia. Int $\mathcal{F}$ Parasitol 1999; 29: 331-9 with a correction in Int f Parasitol 2000; 20: 228

9. Noyes HA, Stevens JR, Teixeira M, Phelan J, Holz P. Corrigendum to "A nested PCR for the ssrRNA gene detects Trypanosoma binneyi in the platypus and Trypanosoma sp. in wombats and kangaroos in Australia." Int 7 Parasitol 2000; 20: 228.

10. Reithinger R, Mohsen M, Wahid M et al. Efficacy of thermotherapy to treat cutaneous leishmaniasis caused by Leishmania tropica in Kabul, Afghanistan: A randomized, control trial. Clin Infect Dis 2005; 40: 1148-55.

11. Bailey MS, Caddy AJ, McKinnon KA et al. Zoonotic cutaneous leishmaniasis with atypical features from Afghanistan. (in press).

12. Herwaldt BL, Berman JD. Recommendations for treating leishmaniasis with sodium stibogluconate (Pentostam) and review of pertinent clinical studies. Am F Trop Med Hyg 1992; 46: 296-306.

13. Aronson NE, Wortmann GW, Johnson SC et al. Safety and efficacy of intravenous sodium stibogluconate in the treatment of leishmaniasis: Recent US military experience. Clin Infect Dis 1998; 27: 1457-64. 
14. Wortmann G, Miller RS, Oster C, Jackson J, Aronson N. A randomized, double-blind study of the efficacy of a 10- or 20-day course of sodium stibogluconate for treatment of cutaneous leishmaniasis in United States military personnel. Clin Infect Dis 2002; 35: 261-7.

15. Seaton RA, Morrison J, Man I, Watson J, Nathwani D. Out-patient parenteral antimicrobial therapy - a viable option for the management of cutaneous leishmaniasis. $O 7 M$ 1999; 92: 659-67.

16. Alrajhi AA, Ibrahim EA, De Vol EB, Khairat $M$, Faris RM, Maguire JH. Fluconazole for the treatment of cutaneous leishmaniasis caused by Leishmania major. N Engl f Med 2002; 346: 891-5.

17. Navin TR, Arana BA, Arana FE, Berman JD, Chajon JF. Placebo-controlled clinical trial of sodium stibogluconate (Pentostam) versus ketoconazole for the treating cutaneous leishmaniasis in Guatemala. F Infect Dis 1992; 165: 528-34.

18. Soto J, Arana BA, Toledo J et al. Miltefosine for New World Cutaneous Leishmaniasis. Clin Infect Dis 2004; 38: 1266-72.

19. Lightburn E, Morand JJ, Meynard JB et al. Management of American cutaneous leishmaniasis: Outcome of 326 cases treated with high-dose pentamidine isethionate. Med Trop (Mars) 2003; 63: 35-44.

20. Andersen EM, Cruz-Saldarriaga M, LlanosCuentas A et al. Comparison of meglumine antimoniate and pentamidine for Peruvian cutaneous leishmaniasis. Am f Trop Med Hyg 2005; 72: 133-7.

\section{Appendix 1.}

Specialists to whom military patients with suspected CL may be referred.

Dr. Chris Ellis

Consultant Physician

The Infectious Diseases Unit

Birmingham Heartlands Hospital

Bordesley Green East

Birmingham

B9 5SS

Phone: 01214240357

Fax: 01214241309

Email: christopher.ellis@heartsol.wmids.nhs.uk

Dr. Tim O'Dempsey

Consultant Physician

Liverpool School of Tropical Medicine

Pembroke Place

Liverpool

L3 5QA

Phone: 01517053109

Fax: 01517053365

Email: tjdod@liv.ac.uk

Dr. Diana Lockwood

Consultant Physician

Hospital for Tropical Diseases

Mortimer Market

Capper Street

London

WC1E 6AU

Phone: 02073879300 ext. 5470

Fax: 02073809761

Email: diana.lockwood@1shtm.ac.uk

\section{Appendix 2.}

Diagnostic laboratories specialising in leishmaniasis.

Dr. Wendi Bailey

The Diagnostic Laboratory

Liverpool School of Tropical Medicine

Pembroke Place

Liverpool

L3 5QA

Phone:0151705 3290

Fax: 01517053365

Email: jwbailey@liv.ac.uk

Prof. Peter Chiodini / Ms. Julie Watson Department of Clinical Parasitology

Hospital for Tropical Diseases

Mortimer Market

Capper Street

London

WC1E 6AU

Phone: 02073879300 ext. 5414

Fax: 02073830041

Email: peter.chiodini@uclh.org julie.watson@uclh.org 


\section{Appendix 3}

Data Collection from Miliatry Cutaneous Leishmaniasis Cases

\begin{tabular}{|l|l|l|l|}
\hline Last Name & & First Name & \\
\hline Service Number & & Military Rank & \\
\hline Age & & Date of Birth & \\
\hline Height & & Weight & \\
\hline Regiment & & Company/Squadron & \\
\hline Platoon/Troop & & Job Description & \\
\hline
\end{tabular}

\begin{tabular}{|l|l|l|l|}
\hline $\begin{array}{l}\text { Countries Visited } \\
\text { (in last 2 years) }\end{array}$ & Areas \& Terrains & $\begin{array}{c}\text { Activities \& } \\
\text { Accommodation }\end{array}$ & Dates \& Duration \\
\hline & & & \\
\hline & & & \\
\hline & & & \\
\hline & & & \\
\hline
\end{tabular}

\begin{tabular}{|l|l|}
\hline Date of arrival in country where CL originated & \\
\hline Date of initial sandfly bite (if recalled) & \\
\hline Date when lesions first noticed & \\
\hline Date when seen by specialist & \\
\hline
\end{tabular}

\begin{tabular}{|l|l|}
\hline Number of skin lesions & \\
\hline Sites of skin lesions (describe) & \\
\hline Sizes of skin lesions (maximum diameters) & \\
\hline Lesions = worsening / static / improving / healed & \\
\hline Secondary infection (organism if known) & \\
\hline Lymph node / lymphatic involvement (describe) & \\
\hline
\end{tabular}

\begin{tabular}{|l|l|}
\hline Aspirate impression smear result & \\
\hline Aspirate PCR results & \\
\hline Scraping impression smear result & \\
\hline Scraping PCR results & \\
\hline Biopsy impression smear result & \\
\hline Biopsy PCR results & \\
\hline Biopsy tissue culture result & \\
\hline Biopsy histology result (granulomata/amastigotes) & \\
\hline
\end{tabular}

Photographs of lesions available

Case notified with F Med 85 (as per SGPL 09/04)

Treatment received + dose + duration $/$ frequency

Date when treatment started

Adverse effects due to treatment

Treatment interruption + reason + date + duration

Maximum FBC abnormalities + date

Maximum U\&E abnormalities + date

Maximum LFT abnormalities + date

Maximum amylase abnormality + date

Maximum ECG abnormalities + date

Date when treatment completed 


\begin{tabular}{|l|l|}
\hline Progress at $6 / 52$ after treatment started & \\
\hline (Progress at $10 / 52$ after treatment started) & \\
\hline (Progress at $14 / 52$ after treatment started) & \\
\hline (Progress at $18 / 52$ after treatment started) & \\
\hline (Progress at 22/52 after treatment started) & \\
\hline Progress at 26/52 after treatment started & \\
\hline
\end{tabular}

\begin{tabular}{|l|l|l|l|l|}
\hline Prevention used in location where CL originated: & $\begin{array}{c}\text { Never } \\
(<10 \%)\end{array}$ & $\begin{array}{c}\text { Sometimes } \\
(10-50 \%)\end{array}$ & $\begin{array}{l}\text { Usually } \\
(50-90 \%)\end{array}$ & $\begin{array}{l}\text { Always } \\
(>90 \%)\end{array}$ \\
\hline Fully clothed from dusk to dawn? & & & & \\
\hline Sleeves rolled down from dusk to dawn? & & & & \\
\hline Hat worn at dusk and dawn? & & & & \\
\hline Clothing dipped in "peripel" every 6 weeks? & & & & \\
\hline Issued insect repellent applied every 8-12 hours? & & & & \\
\hline Non-issued insect repellent used instead? & & & & \\
\hline Slept under bednet? & & & & \\
\hline Bednet dipped in "peripel" in last 6 months? & & & & \\
\hline Knockdown insecticide spray for inside bednet? & & & & \\
\hline Swingfog / environmental spraying used daily? & & & & \\
\hline
\end{tabular}

Additional information : 